\title{
Risk of fragility fracture among patients with gout and the effect of urate-lowering therapy
}

\author{
Alyshah Abdul Sultan PhD, Rebecca Whittle MSc, Sara Muller PhD, Edward Roddy PhD, Christian D. Mallen PhD, \\ Milica Bucknall PhD, Toby Helliwell PhD, Samantha Hider PhD, Zoe Paskins PhD
}

Cite as: CMAJ 2018 May 14;190:E581-7. doi: 10.1503/cmaj.170806

\begin{abstract}
BACKGROUND: Previous studies that quantified the risk of fracture among patients with gout and assessed the potential effect of urate-lowering therapy have provided conflicting results. Our study aims to provide better estimates of risk by minimizing the effect of selection bias and confounding on the observed association.
\end{abstract}

METHODS: We used data from the Clinical Practice Research Datalink, which records primary care consultations of patients from across the United Kingdom. We identified patients with incident gout from 1990 to 2004 and followed them up until 2015. Each patient with gout was individually matched to 4 controls on age, sex and general practice. We calculated absolute rate of fracture and hazard ratios (HRs) using Cox regression models. Among patients with gout, we assessed the impact of urate-lowering therapy on fracture, and used landmark analysis and propensity score matching to account for immortal time bias and confounding by indication.

RESULTS: We identified 31781 patients with incident gout matched to 122961 controls. The absolute rate of fracture was similar in both cases and controls (absolute rate $=53$ and 55 per 10000 person-years, respectively) corresponding to an HR of 0.97 (95\% confidence interval 0.92-1.02). Our finding remained unchanged when we stratified our analysis by age and sex. We did not observe statistically significant differences in the risk of fracture among those prescribed urate-lowering therapy within 1 and 3 years after gout diagnosis.

INTERPRETATION: Overall, gout was not associated with an increased risk of fracture. Urate-lowering drugs prescribed early during the course of disease had neither adverse nor beneficial effect on the long-term risk of fracture.

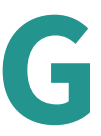
out is the most common type of inflammatory arthritis, affecting $2.4 \%$ of adults in the United Kingdom. ${ }^{1}$ It has been hypothesized that, in common with other chronic inflammatory arthritides such as rheumatoid arthritis and spondyloarthropathy, ${ }^{2,3}$ gout may be associated with an increased risk of fracture, primarily owing to the negative effects of chronic inflammation on bone, because proinflammatory cytokines are known to induce bone loss. However, the effects of serum urate on bone health are still under debate ${ }^{4,5}$ and previous studies that have assessed the impact of gout and urate-lowering therapy on fracture risk have provided conflicting results. For instance, a population-based study from Taiwan ${ }^{6}$ found a $17 \%$ increased risk of fracture among patients with gout and reported lower fracture risk among those prescribed urate-lowering therapy. In contrast, a registry-based study from Denmark $^{7}$ found a $9 \%$ higher risk of fracture among patients prescribed allopurinol compared with patients who were not. A study in the United States ${ }^{8}$ concluded that gout has no effect on the risk of nonvertebral fracture. Two of the studies were based on large administrative data ${ }^{6,7}$ but failed to take into account lifestyle-related factors such as body mass index (BMI) and alcohol consumption; they also did not adequately address selection bias associated with potentially delayed urate-lowering therapy after diagnosis, ${ }^{9}$ which may have affected their overall conclusions. Fragility fractures are associated with increased cost of health care ${ }^{10,11}$ and a substantial cause of morbidity. Therefore, the aim of this study was to quantify precisely the risk of fracture among patients with gout in the UK and assess the potential effect of urate-lowering therapy on fracture risk estimates using a large population-based primary health care database.

\section{Methods}

\section{Data source, design and setting}

We used the Clinical Practice Research Datalink, ${ }^{12}$ a large database containing UK primary care medical records of anonymized patients (supplementary data, section on Clinical Practice Research Datalink [Appendix 1, available at www.cmaj.ca/lookup/suppl/doi:10.1503/ cmaj.170806/-/DC1]). The database is representative of the general UK population in terms of age, sex, ethnicity and lifestyle-related characteristics. ${ }^{13,14}$ We identified individuals with a first-ever recorded Read code diagnosis of gout from general practices between 1990 
and 2004, who were then followed up until 2015. The diagnosis of gout was based on a medical code assigned by the physician, which has been previously validated in the Clinical Practice Research Datalink and has a positive predictive value of $90 \% .{ }^{15}$ Each patient was assigned an index date that corresponded to the date of their diagnosis of gout and randomly matched to 4 controls, who did not have a diagnosis of gout or evidence of urate-lowering therapy, who were registered at the same practice and were alive and contributing data at the index date. They were matched on age ( $\pm 3 \mathrm{yr}$ ) and sex. Controls were assigned the same index date as their matched gout case. For both cases and controls, follow-up commenced from the index date. Those with a history of fragility fracture, less than 1 year of follow-up before the index date or less than 3 years of follow-up after index date were excluded from the study.

The event of interest was time from the index date until the first diagnosis of fracture. Medical codes for fractures at sites of major osteoporotic fracture were selected (vertebrae, humerus, wrist and hip) in addition to codes for fragility fractures of unspecified site. For the purpose of this study, we were interested only in the incidence of first fracture; thus, all subsequent fracture events were ignored. Van Staa and colleagues ${ }^{16}$ carried out external validation of fracture diagnosis in the Clinical Practice Research Datalink and found that $88 \%$ and $91 \%$ of vertebral and hip fracture diagnoses, respectively, were verified by physicians.

For each individual in our study, we extracted information on relevant lifestyle-related characteristics (smoking status and alcohol consumption), BMI and comorbidities (defined using the Charlson index). ${ }^{17}$ We also extracted information on selected medication use (antihypertensive agents, antidiabetic agents, opioids, glucocorticoids, proton pump inhibitors, selective serotonin reuptake inhibitors and bisphosphonates) and history of falls. We ascertained information on comorbidities and lifestyle-related characteristics within 5 years, and on medication use within 1 year before the index date. As the timing of beginning urate-lowering therapy varies after diagnosis of gout, we used landmark analysis to examine the effect of urate-lowering therapy on the risk of first fracture among patients with gout. ${ }^{18}$ This method deals with immortal time bias, which biases the results in favour of the treatment under study, by granting a false survival advantage to the treated group. In landmark analysis, ${ }^{19}$ a fixed time after the start of therapy is selected for conducting survival analysis (Figure 1). Only patients who were alive and contributing data at landmark time were included in the analysis. The exposure (urate-lowering therapy) was evaluated between the index date and the landmark time, whereas a fracture event was considered only after the landmark time point. Two landmark points were considered in the analysis ( 1 and $3 \mathrm{yr}$ after diagnosis), based on a previously published study. ${ }^{20}$ Only patients who were prescribed more than 6 months of urate-lowering therapy were considered to be exposed.

\section{Statistical analysis}

We calculated the incidence of fracture as the number of first recorded fractures per 10000 person-years. Using a Cox regression model, we calculated hazard ratios (HRs) and 95\% confidence intervals (Cls), comparing the risk of fracture between gout cases and controls adjusted for various covariates. We accounted for clustering by practice by using robust standard errors. We imputed missing values of BMI by multiple imputation using chained equations. The proportional hazard assumption was tested using Schoenfeld residuals. We stratified our analysis by age, sex and fracture site. To assess the effect of urate-lowering therapy on fracture risk among those with gout, we used propensity score matching methods to account for confounding by indication. The propensity score for urate-lowering therapy represents the probability that a patient is prescribed the therapy for at least 6 months during the exposure window, given their observed covariates (described further in Appendix 1). A logistic regression model was used to estimate propensity scores; subsequently, each patient with gout who was exposed to urate-lowering therapy was matched to 1 patient with gout who had not been exposed to the therapy, based on their propensity score with caliper distance of $0.2 .{ }^{19}$ We used a greedy algorithm to select matches: i.e., we selected the closest matching first, then the closest remaining matching, until there were no acceptable matches. We separately compared the risk of fracture among those who received more than 6 months of urate-lowering therapy within a 1- and 3-year exposure window compared with their matched controls who had not been exposed to urate-lowering therapy during that period, using a Cox regression model. We conducted all analyses using Stata version 14.

\section{Ethics approval}

This study was approved by the Clinical Practice Research Datalink Independent Scientific Advisory Committee (reference number 15 165RA).

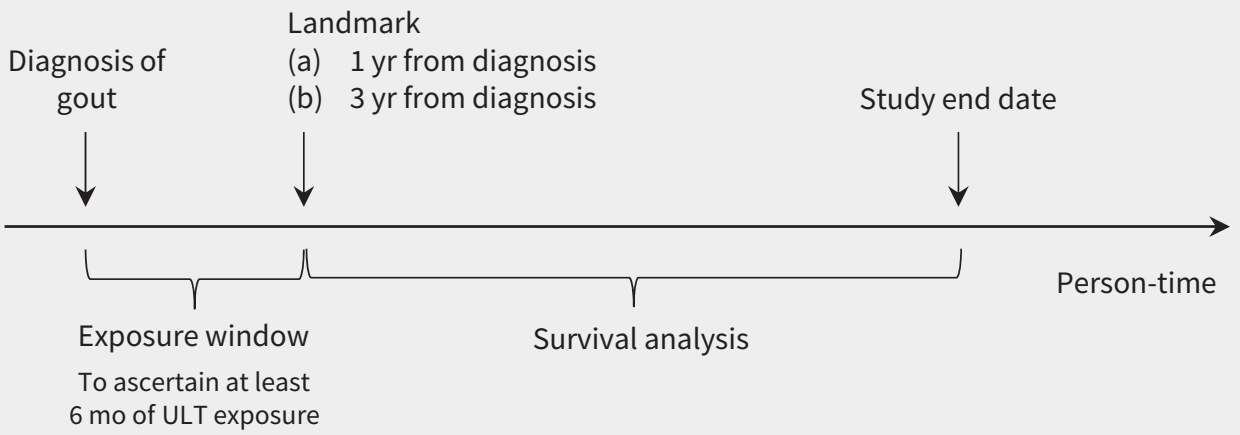

Figure 1: Graphical representation of landmark analysis. Note: ULT = urate-lowering therapy. 


\section{Results}

We identified 31781 cases of patients with incident gout who were matched to 122961 controls. The median follow-up for our study was 10.8 years (interquartile range [IQR] 6.8-13.6 yr). The characteristics of the study population are summarized in
Table 1. A total of 8934 patients sustained a first fragility fracture at some point during the follow-up period. The absolute rate of fracture among gout cases and controls was 53 and 55 per 10000 person-years, respectively (Table 2). Compared with controls, we found no excess risk of fracture among patients with gout (HR 0.97, 95\% Cl 0.92-1.02). These findings remained consistent

\begin{tabular}{|c|c|c|c|}
\hline Characteristic & $\begin{array}{c}\text { No. }(\%) \text { of controls } \\
n=122961\end{array}$ & $\begin{array}{c}\text { No. (\%) of cases of gout } \\
\qquad n=31781\end{array}$ & $\begin{array}{c}\text { Standardized } \\
\text { difference }\end{array}$ \\
\hline Age, yr; mean \pm SD & $63.1 \pm 12.2$ & $63.5 \pm 12.5$ & 0.04 \\
\hline Male sex & $89978(73.2)$ & $23180(72.9)$ & 0.00 \\
\hline Follow-up time, yr; median (IQR) & $10.8(6.8-13.6)$ & $10.8(6.7-13.4)$ & 0.03 \\
\hline \multicolumn{4}{|l|}{ BMI, $\mathrm{kg} / \mathrm{m}^{2}$} \\
\hline Normal (18.5-24.9) & $34319(27.9)$ & $5773(18.2)$ & 0.41 \\
\hline Underweight (<18.5) & $1151(0.9)$ & $129(0.4)$ & \\
\hline Overweight (25.0-29.9) & $37142(30.2)$ & $11641(36.6)$ & \\
\hline Obese $(\geq 30)$ & $14852(12.1)$ & $7597(23.9)$ & \\
\hline Missing & $35497(28.9)$ & $6641(20.9)$ & \\
\hline \multicolumn{4}{|l|}{ Smoking status } \\
\hline Never smoked or former smoker & $100109(81.4)$ & $26978(84.9)$ & 0.09 \\
\hline Current smoker & $22852(18.6)$ & $4803(15.1)$ & \\
\hline \multicolumn{4}{|l|}{ Alcohol consumption, units per wk } \\
\hline Never consumed or former drinker & $15143(12.3)$ & $3632(11.4)$ & 0.30 \\
\hline Current 1-9 & $50568(41.1)$ & $12181(38.3)$ & \\
\hline Current $\geq 10$ & $23819(19.4)$ & $9909(31.2)$ & \\
\hline Unknown & $33431(27.2)$ & $6059(19.1)$ & \\
\hline History of falls & $3558(2.9)$ & $1143(3.6)$ & 0.04 \\
\hline \multicolumn{4}{|l|}{ Charlson index } \\
\hline 0 & $96284(78.3)$ & $22419(70.5)$ & 0.19 \\
\hline $1-2$ & $23377(19.0)$ & $7872(24.8)$ & \\
\hline $3-4$ & $2885(2.3)$ & $1231(3.9)$ & \\
\hline$\geq 5$ & $415(0.3)$ & $259(0.8)$ & \\
\hline \multicolumn{4}{|l|}{ Medications } \\
\hline Glucocorticoids & $3931(3.2)$ & $1506(4.7)$ & 0.08 \\
\hline Opioids & $11269(9.2)$ & $4658(14.7)$ & 0.17 \\
\hline Bisphosphonates & $556(0.5)$ & $125(0.4)$ & 0.01 \\
\hline SSRIS & $3979(3.2)$ & $1122(3.5)$ & 0.02 \\
\hline Statins & $8811(7.2)$ & 3999 (12.6) & 0.18 \\
\hline Antihypertensive agents & $29634(24.1)$ & $14233(44.8)$ & 0.44 \\
\hline Antidiabetic agents & $4894(4.0)$ & $1173(3.7)$ & 0.02 \\
\hline PPIs & $8339(6.8)$ & $3366(10.6)$ & 0.14 \\
\hline NSAIDs & $18892(15.4)$ & $22264(70.1)$ & 1.33 \\
\hline ASA & $14141(11.5)$ & $5735(18)$ & 0.19 \\
\hline \multicolumn{4}{|c|}{$\begin{array}{l}\text { Note: } A S A=\text { acetylsalicylic acid, } B M I=\text { body mass index, IQR = interquartile range, NSAID = nonsteroidal anti-inflammatory drug, } \\
P P I=\text { proton pump inhibitor, } S D=\text { standard deviation, } S S R I=\text { selective serotonin reuptake inhibitor. } \\
\text { *Unless otherwise specified. } \\
\text { tStandardized difference = difference in means or proportion divided by standard error; imbalance defined as absolute value } \\
\text { greater than } 0.20 \text { (small effect size). }\end{array}$} \\
\hline
\end{tabular}


when we stratified our analysis by age. Although women had higher absolute risk of fracture than men, their excess risk compared with their matched controls was not statistically significant (HR 0.96, 95\% Cl 0.89-1.02). Compared with controls, patients with gout had no increased risk of vertebral or nonvertebral fractures (Supplementary Table 1, Appendix 1). For our 1-year landmark analysis, we included 31668 patients with incident gout (Figure 2) who did not die, transfer out of the practice or have a fracture within the exposure window. The baseline characteristics of patients who were exposed and those who were not exposed to urate-lowering therapy within 1 year after gout diagnosis are summarized in Supplementary Table 2 (Appendix 1). After propensity score matching, we found no difference in the baseline characteristics by status of exposure to urate-lowering therapy, which shows the quality of our matching (Supplementary Table 3, Appendix 1). There was no difference in the risk of long-term fracture among those who were exposed and were not exposed to at least 6 months of urate-lowering therapy within a year of their diagnosis of gout (HR 1.01, 95\% Cl 0.84-1.22) (Table 3). We also observed similar findings in our 3-year landmark analysis.

\section{Interpretation}

Using data from a large, nationally representative cohort, we compared the risk of fragility fracture (composite of vertebral and nonvertebral fracture) observed among patients with incident gout to the general population. Overall, gout was not associated with an increased risk of vertebral or nonvertebral facture. These findings were consistent when we stratified our analysis by age and sex. Among those with incident gout, we found that having at least 6 months of urate-lowering therapy within 1 and 3 years of diagnosis had neither adverse nor beneficial effect on the long-term risk of fragility fractures compared with receiving no or less than 6 months of urate-lowering therapy.
We found no association between gout and the risk of fragility fracture. This finding is in contrast to a Taiwanese study that reported a statistically significant $17 \%$ higher risk of fracture among patients with gout compared with their matched controls. ${ }^{6}$ This may be a result of the difference in the study population and outcome definition used. For instance, our study focused primarily on fragility fractures, whereas the Taiwanese study included all types of fractures, including fracture of ankle or foot, which accounted for $15 \%$ of all fractures in that study's gout cohort and had the largest excess risk (34\%). The neutral effect of gout remained when we stratified our analysis by fracture site. This finding is consistent with other studies. ${ }^{6,8}$ Although Tzeng and colleagues ${ }^{6}$ found a $14 \%$ increased risk of vertebral fracture, their study failed to take into account important lifestyle-related factors (BMI, smoking status and alcohol consumption), which may have confounded their finding.

Although women are more likely to sustain fragility fractures than men, few studies provide risk estimates by sex..$^{6-8} \mathrm{~A}$ US-based study $^{21}$ reported positive associations between gout and incidence of hip and wrist fracture in women, with an adjusted excess risk of $12 \%$ and $38 \%$, respectively. Although this study refutes our findings, it used self-reported information on both gout and fracture, which may have introduced bias. Furthermore, their study findings may not be generalizable to the wider population, because they were based exclusively on a cohort of nurses.

We found that urate-lowering therapy had neither a beneficial nor adverse effect on the long-term risk of fragility fracture. The existing literature on the topic is conflicting ${ }^{6,7,22}$ and, unlike our study, does not address the issues of immortal time bias or confounding by indication. For instance, Dennison and colleagues ${ }^{7}$ reported $9 \%$ excess risk of osteoporotic fractures among those prescribed allopurinol compared with nonusers. Although these authors used propensity score matching, there were still significant differences in the baseline characteristics and comorbidities between groups that were exposed and not exposed. Moreover, their controls may have included

Table 2: Absolute and relative rate of fragility fracture among cases compared with controls*

\begin{tabular}{|c|c|c|c|c|c|c|}
\hline \multirow[b]{2}{*}{ Characteristic } & \multicolumn{2}{|r|}{ Unexposed } & \multicolumn{2}{|r|}{ Exposed } & \multirow[b]{2}{*}{ Unadjusted HR (95\% CI) } & \multirow[b]{2}{*}{ Adjusted HR $(95 \% \mathrm{Cl})$} \\
\hline & $n$ & Rate† $(95 \% \mathrm{CI})$ & $n$ & Rate $\neq(95 \% \mathrm{CI})$ & & \\
\hline Overall & 7164 & $54.7(53.5-56.0)$ & 1770 & $52.9(50.5-55.6)$ & $0.97(0.92-1.02)$ & $0.95(0.89-1.01)$ \\
\hline \multicolumn{7}{|c|}{ Age, yr (quartile) } \\
\hline 1 & 683 & $18.3(16.9-19.7)$ & 179 & $18.6(16.1-21.6)$ & $1.02(0.86-1.20)$ & $0.90(0.73-1.10)$ \\
\hline 2 & 1147 & $32.2(30.3-34.0)$ & 296 & $32.7(29.2-36.7)$ & $1.01(0.90-1.16)$ & $0.92(0.79-1.07)$ \\
\hline 3 & 2020 & $61.7(59.1-64.5)$ & 505 & $61.7(56.6-67.4)$ & $1.02(0.92-1.12)$ & $1.01(0.91-1.14)$ \\
\hline 4 & 3314 & $132.4(127.9-136.9)$ & 790 & $119.6(111.6-128.3)$ & $0.92(0.85-1.00)$ & $0.94(0.86-1.03)$ \\
\hline \multicolumn{7}{|l|}{ Sex } \\
\hline Male & 3016 & $30.9(29.4-32.0)$ & 793 & $31.5(29.4-33.8)$ & $1.02(0.94-1.10)$ & $0.99(0.90-1.09)$ \\
\hline Female & 4148 & $124.7(121.0-128.5)$ & 977 & $117.7(110.5-125.3)$ & $0.96(0.89-1.02)$ & $0.94(0.87-1.01)$ \\
\hline
\end{tabular}


patients who did not have gout, which may have affected their observed association. In contrast, Tzeng and colleagues ${ }^{6}$ reported $28 \%$ lower risk of fracture among patients with gout who were prescribed urate-lowering therapy compared with those who were not prescribed the therapy. However, this study overlooked that patients receiving urate-lowering therapy must be event-free from the time of gout diagnosis to the time of the first prescription of urate-lowering therapy be considered exposed, whereas no such requirement is necessary for the unexposed group.

\section{Strengths and limitations}

Using data from primary care in the UK, we conducted one of the largest studies with more than 25 years of follow-up to quantify the occurrence of fragility fracture among patients with gout compared with a matched group of individuals without gout in a contemporary population-based manner. Our use of a nationally representative cohort should enable our study findings to be generalizable not only to the UK but also to other developed countries with similar health care systems. Furthermore, the

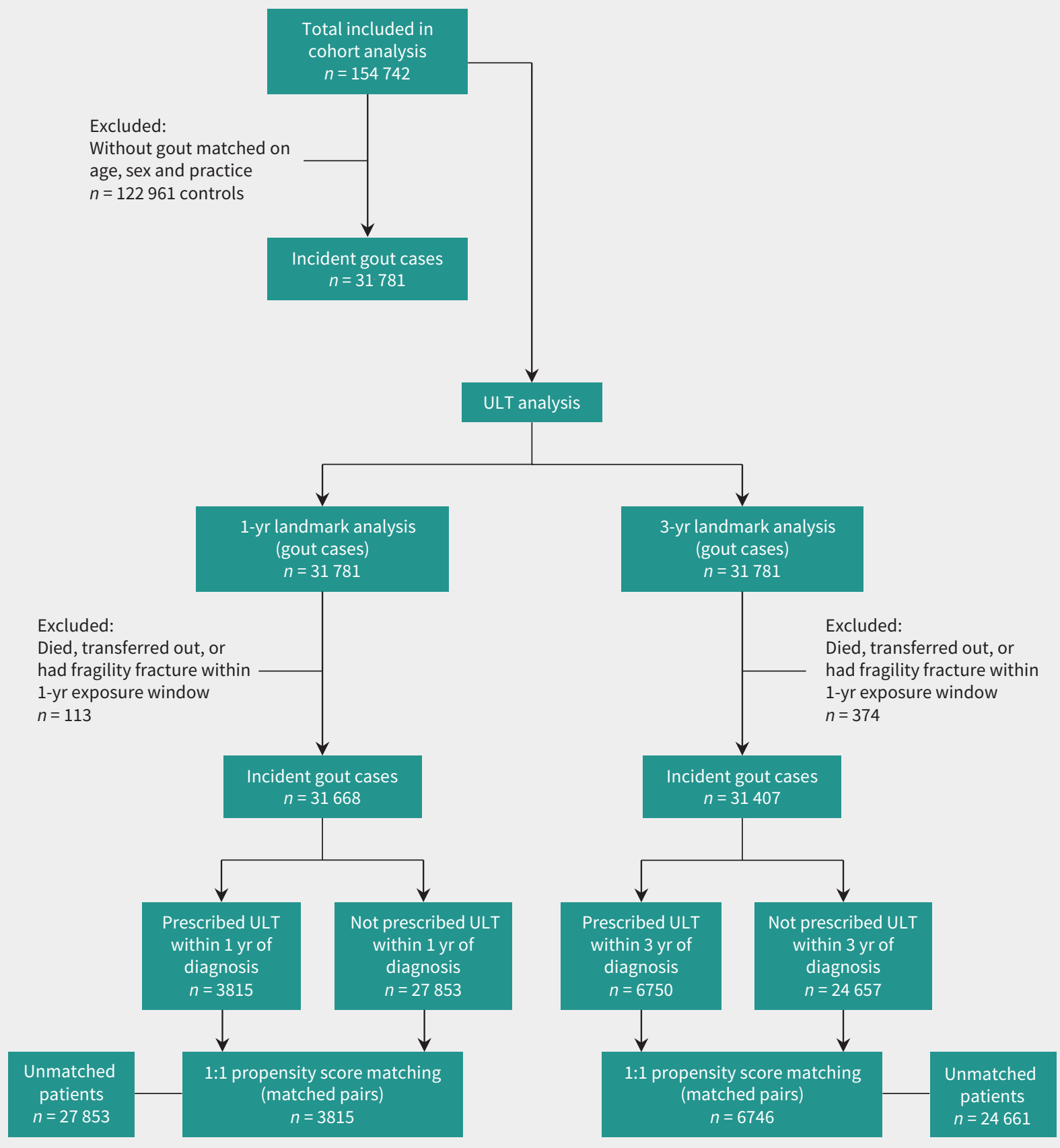

Figure 2: Flow diagram of study population. Note: ULT = urate-lowering therapy. 
Table 3: Risk of fracture at 1- and 3-year landmark (propensity score-matched)

1-year landmark analysis

ULT not

prescribed rate*

$(95 \% \mathrm{Cl})$

Characteristic

Overall

Vertebral

Nonvertebral fracture

Wrist

Hip

Humerus
$62.0(54.3-70.8)$

$8.3(5.8-12.0)$

$47.1(40.4-54.9)$

$19.2(15.1-24.4)$

$18.4(14.4-23.5)$

$9.5(6.5-13.3)$
ULT prescribed rate $(95 \% \mathrm{Cl})$

$62.7(55.0-71.6)$

$7.7(3.7-8.8)$

$51.9(44.9-60.0)$

$21.1(16.8-26.5)$

$23.1(18.6-28.7)$

$7.7(5.3-11.2)$
ULT not

prescribed rate

$(95 \% \mathrm{Cl})$ 3-year landmark analysis

\section{ULT prescribed rate $(95 \% \mathrm{Cl})$}

HR $(95 \% \mathrm{Cl})$

$1.01(0.84-1.22)$

$0.68(0.39-1.20)$

$65.3(58.5-72.8)$

$65.2(58.5-72.7)$

$1.00(0.85-1.16)$

$9.0(6.7-12.1)$

$6.5(4.6-9.1)$

$0.71(0.45-1.12)$

$1.10(0.89-1.36)$

$50.6(44.7-57.3)$

$52.1(46.1-58.9)$

$1.03(0.87-1.22)$

$1.10(0.79-1.53)$

17.9 (14.6-22.1)

$18.0(14.6-22.1)$

$1.00(0.74-1.34)$

$1.25(0.90-1.75)$

$24.3(20.3-29.0)$

$25.2(21.2-30.1)$

$1.04(0.81-1.34)$

$0.81(0.49-1.35)$

$8.90(6.6-11.4)$

$1.06(0.70-1.62)$

Note: $\mathrm{Cl}=$ confidence interval, $\mathrm{HR}=$ hazard ratio, ULT = urate-lowering therapy

*Per 10000 person-years.

prospective nature of the data recording enables us to understand better the temporal relationship between exposure and outcome with minimum bias.

However, our study has several limitations. Our reliance on physicians to make the diagnosis of gout, rather than using the gold standard of visualization of monosodium urate crystals in joint fluid or identification of tophi on examination, could have led to misclassification. However, the diagnosis of gout has been previously validated in the Clinical Practice Research Datalink with high accuracy: ${ }^{15}$ it is therefore unlikely that there is any major error in our findings resulting from misclassification of our cases. The findings of this validation study are consistent with another study in which $83 \%$ of cases of gout diagnosed by general practitioners were independently validated by a rheumatologist on clinical grounds. ${ }^{23}$ Although this previous study found a high positive predictive value, it did not give an indication of negative predictive value (or sensitivity), and there is a possibility of missing cases that may have been diagnosed in a specialized setting. However, this is unlikely, because gout is managed principally in primary care. Furthermore, a recent study using a similar database has shown higher prevalence of gout ${ }^{1}$ than previously reported. Therefore, we believe it is unlikely that there is any major error in our findings resulting from misclassification of our cases.

Similar misclassification may also exist for fracture diagnosis, although recording of fracture has also been validated in the Clinical Practice Research Datalink; ${ }^{16}$ differential recording in the diagnosis of fracture among cases and controls is unlikely. Finally, the use of 1- and 3-year landmarks for our urate-lowering therapy analysis means that our findings can be generalized only to those who were alive and contributing data at those landmark points, who did not develop fracture within the exposure window and who were prescribed at least 6 months of urate-lowering therapy after their initial diagnosis of gout.

\section{Conclusion}

We found no excess risk of fragility fractures among patients with gout. Our findings remained consistent when we stratified our analysis by age, sex and fracture site. Our propensity score-matched landmark analyses showed that prescription of at least 6 months of urate-lowering therapy within 1 and 3 years of patients' initial diagnosis of gout had neither beneficial nor adverse effects on long-term risk of fracture. These findings should be reassuring to patients, health care policymakers and clinicians.

\section{References}

1. Kuo CF, Grainge MJ, Mallen C, et al. Rising burden of gout in the UK but continuing suboptimal management: a nationwide population study. Ann Rheum Dis 2015;74:661-7.

2. van Staa TP, Geusens P, Bijlsma JW, et al. Clinical assessment of the long-term risk of fracture in patients with rheumatoid arthritis. Arthritis Rheum 2006;54:3104-12.

3. Vosse $D$, Landewé R, van der Heijde $D$, et al. Ankylosing spondylitis and the risk of fracture: results from a large primary care-based nested case-control study. Ann Rheum Dis 2009;68:1839-42.

4. Mehta T, Bůžková P, Sarnak MJ, et al. Serum urate levels and the risk of hip fractures: data from the Cardiovascular Health Study. Metabolism 2015;64:438-46.

5. Kim BJ, Baek S, Ahn SH, et al. Higher serum uric acid as a protective factor against incident osteoporotic fractures in Korean men: a longitudinal study using the National Claim Registry. Osteoporos Int 2014;25:1837-44.

6. Tzeng HE, Lin CC, Wang IK, et al. Gout increases risk of fracture: a nationwide population-based cohort study. Medicine (Baltimore) 2016;95:e4669.

7. Dennison EM, Rubin $\mathrm{KH}$, Schwarz P, et al. Is allopurinol use associated with an excess risk of osteoporotic fracture? A National Prescription Registry study. Arch Osteoporos 2015;10:36.

8. Kim SC, Paik JM, Liu J, et al. Gout and the risk of non-vertebral fracture. J Bone Miner Res 2017;32:230-6.

9. Lévesque LE, Hanley JA, Kezouh A, et al. Problem of immortal time bias in cohort studies: example using statins for preventing progression of diabetes. BMJ 2010;340:b5087.

10. Leal J, Gray AM, Prieto-Alhambra D, et al. Impact of hip fracture on hospital care costs: a population-based study. Osteoporos Int 2016;27:549-58.

11. Effective secondary prevention of fragility fractures: clinical standards for fracture liaison services. Camerton (UK): National Osteoporosis Society; 2015. Available: https://staging.nos.org.uk/media/1776/clinical-standards-report.pdf (accessed 2017 Oct. 20).

12. Clinical Practice Research Datalink [main page]. London (UK): CPRD. Available: www.cprd.com/intro.asp (accessed 2017 Nov. 29).

13. Crooks C. Epidemiology of upper gastrointestinal bleeding studying its causes and outcomes using case control studies and surivival analyses [PhD thesis]. Nottingham (UK): University of Nottingham; 2013. 
14. Herrett E, Gallagher AM, Bhaskaran K, et al. Data resource profile: Clinical Practice Research Datalink (CPRD). Int J Epidemiol 2015;44:827-36.

15. Meier CR, Jick H. Omeprazole, other antiulcer drugs and newly diagnosed gout. $\mathrm{Br} J$ Clin Pharmacol 1997;44:175-8.

16. Van Staa TP, Abenhaim L, Cooper C, et al. The use of a large pharmacoepidemiological database to study exposure to oral corticosteroids and risk of fractures: validation of study population and results. Pharmacoepidemiol Drug Saf 2000;9:359-66.

17. Charlson ME, Pompei $\mathrm{P}$, Ales $\mathrm{KL}$, et al. A new method of classifying prognostic comorbidity in longitudinal studies: development and validation. J Chronic Dis 1987;40:373-83.

18. Dafni U. Landmark analysis at the 25-year landmark point. Circ Cardiovasc Qual Outcomes 2011;4:363-71.
19. Giobbie-Hurder A, Gelber RD, Regan MM. Challenges of guarantee-time bias. J Clin Oncol 2013;31:2963-9.

20. Kuo CF, Grainge MJ, Mallen C, et al. Effect of allopurinol on all-cause mortality in adults with incident gout: propensity score-matched landmark analysis. Rheumatology (Oxford) 2015;54:2145-50.

21. Paik JM, Kim SC, Feskanich D, et al. Gout and risk of fracture in women: a prospective cohort study. Arthritis Rheumatol 2017;69:422-8.

22. Basu U, Goodbrand J, McMurdo ME, et al. Association between allopurinol use and hip fracture in older patients. Bone 2016;84:189-93.

23. Roddy E, Zhang W, Doherty M. Concordance of the management of chronic gout in a UK primary-care population with the EULAR gout recommendations. Ann Rheum Dis 2007;66:1311-5.

\section{Competing interests: None declared.}

This article has been peer reviewed.

Affiliations: Arthritis Research UK Primary Care Centre (Abdul Sultan, Whittle, Muller, Roddy, Mallen, Bucknall, Helliwell, Hider, Paskins), Research Institute for Primary Care \& Health Sciences, Keele University, Staffordshire, UK; Haywood Academic Rheumatology Centre (Roddy, Hider, Paskins), Staffordshire and Stoke-on-Trent Partnership Trust, Stokeon-Trent, UK

Contributors: Zoe Paskins, Edward Roddy, Christian Mallen, Samantha Hider, Sara Muller and Milica Bucknall contributed to the conception and design of the study. Sara Muller, Rebecca Whittle and Alyshah Abdul Sultan contributed to the acquisition of data. Alyshah Abdul Sultan, Rebecca Whittle and Zoe Paskins were responsible for analyzing and interpreting the data. Alyshah Abdul Sultan and Rebecca Whittle contributed to the statistical analysis. All of the authors drafted the manuscript, revised it critically for important intellectual content, gave final approval of the version to be published and agreed to be accountable for all aspects of the work.

Funding: This study was funded by the National Institute for Health Research (NIHR) School for Primary Care Research (grant number 259). Christian Mallen is funded by the NIHR Collaborations for Leadership in Applied Health Research and Care West Midlands, the NIHR School for Primary Care Research and a
NIHR Research Professorship in General Practice, which also supports Alyshah Abdul Sultan (NIHR-RP-2014-04-026). Toby Helliwell is funded by an NIHR Clinical Lectureship in General Practice. The views expressed are those of the author(s) and not necessarily those of the National Health Service, the NIHR or the Department of Health. The funder was not involved in the study design; in the collection, analysis and interpretation of data; in the writing of the report; or in the decision to submit the article for publication.

Accepted: Dec. 18, 2017

Correspondence to: Zoe Paskins, z.paskins@keele.ac.uk 